\title{
Collective dynamics in noble-gas and other very simple classical fluids
}

\author{
U.Bafile ${ }^{1}$, F.Barocchi ${ }^{2,3}$, E.Guarini ${ }^{2,3}$ \\ ${ }^{1}$ Istituto dei Sistemi Complessi, Consiglio Nazionale delle Ricerche, via Madonna del Piano 10, I-50019 Sesto \\ Fiorentino, Italy \\ 2 Dipartimento di Fisica, Università di Firenze, via G. Sansone 1, I-50019 Sesto Fiorentino, Italy \\ 3 CNR-INFM CRS-Soft c/o Dipartimento di Fisica, Università di Firenze, via G. Sansone 1, I-50019 Sesto \\ Fiorentino, Italy
}

Received November 12, 2007

\begin{abstract}
Rare gases and their liquids are the simplest systems to study for accurate investigations of the collective dynamics of fluid matter. Much work has been done using different spectroscopic techniques, moleculardynamics simulations, and theoretical developments, in order to gain insight into the microscopic processes involved, in particular, in the propagation of acoustic excitations in gases and liquids. Here we briefly review the interpretation schemes currently applied to the characterization of such excitations, and recall a few results obtained from the analysis of rare-gas fluids and other very simple systems.
\end{abstract}

Key words: dynamics of fluids, collective excitations

PACS: $61.20 . L c, 61.20 . G y, 61.25 . B i$

\section{Introduction}

Rare-gas fluids are the simplest systems, and therefore ideal candidates, for studying collective dynamical properties at a nanoscopic level.

In dealing with such phenomena one would naturally like to focus on the translational motions of the constituent particles. However, even in the most simple molecules there exist complicated static and dynamic correlations due to the rotational and vibrational degrees of freedom. In fact, even when internal and centre-of-mass motions take place in well-separated energy windows, as it happens for light molecules, they generally are not independent of each other. If the dynamics is investigated by means of a spectroscopic probe sensitive to the positions of all atoms of the system, the detected signal includes contributions from all intra- and intermolecular correlations, weighted by cross-section factors that are specific to the employed technique. This fact usually makes the data analysis a difficult task. On the contrary, due to the absence of a molecular structure, monatomic fluids have the nice property that the position of one particle changes with time only because of its translational displacement in the environment of the other ones.

That a deep connection exists between dynamical properties and the forces acting at the atomic level is an obvious fact, but how such a relationship is particularly working is still a fundamental, unanswered question. Molecular-dynamics (MD) computer simulations can be used to try to understand which dynamical behaviour is caused by a given interaction potential model [1-4]. Moreover, if realistic and well-modelled interatomic forces are available, the combination of MD and experimental studies can also shed light on whether specific properties of the true interaction law can be inferred from an actually observed dynamical behaviour.

In this respect, rare gases offer a clear advantage as well. Their interatomic pair potentials are very well known functions of the only variable involved, the distance between the two atoms of a pair [5]. The fundamental assumption that such functions do not depend on the thermodynamic state of the fluid, never contradicted by experimental evidence, has also made possible accurate investigations of interatomic forces in dilute gases using a variety of methods. The total potential 
energy of a dense many-body system can then be appropriately described by assuming that small irreducible contributions due to triplets, quadruplets, and so on, can be added to the dominant pair-additive part. The situation is very different for most molecular systems, whose intermolecular interactions are either unknown or at least not yet well tested with a sufficient accuracy, even at the pair level. Moreover, with the exception of very few cases, the anisotropic part of the potential plays a non-negligible role, making the interaction energy a multi-variable function of distances and orientation angles.

Although the metallic liquids are also simple monatomic systems, the presence of nearly free electrons clearly affects the properties of the interaction between atoms [6], making the modelling of potentials by far a harder task than in insulating fluids. As a result, liquid-metal interaction energies are not yet known with an accuracy comparable to that of the rare-gas case.

Thus, a theoretical or simulation approach to dynamical problems is far easier in noble-gas fluids. These, moreover, are often simpler systems from the experimental point of view. For example, they are easier samples to handle for lack of chemical reactivity, no need of high temperatures, and usually, at least with liquids, of high pressures.

The above facts have made rare gases and liquids one of the preferred choices for the study of microscopic collective dynamics. In this paper, where we focus on very simple systems, we shall obviously be concerned with rare-gas fluids, but we shall also include as examples the case of a simple molecular liquid and that of a rare-gas binary mixture. Liquid metals [7], on the contrary, will not be dealt with here.

We will not consider the important case of quantum fluids. Collective excitations in liquid helium constitute a well-established research field, which would deserve by itself a wholly dedicated treatment $[8,9]$. Hydrogen and deuterium have also been the subject of several investigations [10-17]. Their molecular nature is not an obstacle due to the separability of the centre-of-mass translational dynamics from the rotovibrational motions [18]. However, $\mathrm{H}_{2}$ and, to a lesser extent, $\mathrm{D}_{2}$ also display quantum behaviour in the liquid phases and will not be treated here. Neon, instead, features a modest quantum behaviour and has been investigated using essentially the same approach as the one used for truly classical fluids.

In this paper we restrict ourselves to considering collective acoustic excitations, related to the density fluctuations. The fundamental quantity that reflects this dynamical behaviour is the dynamic structure factor $S(Q, \omega)$, where $Q$ and $\omega$ are the wavevector and frequency of the excitation, respectively. $S(Q, \omega)$ is the time Fourier transform of the intermediate scattering function $F(Q, t)$, which represents the time autocorrelation of the spatial Fourier component with wave vector $Q$ of the microscopic local density $n(\mathbf{r}, t)=\sum_{\alpha=1}^{N} \delta\left(\mathbf{r}-\mathbf{R}_{\alpha}(t)\right)$ [19] for a system of $N$ identical particles at positions $\mathbf{R}_{\alpha}(t)$. Therefore, $S(Q, \omega)$ or $F(Q, t)$ are the right quantities to determine, by means of various methods, in order to gain direct access to the atomic motions in a many-body system.

MD simulation has found in the dynamics of liquids an extremely important field of application [20], also due to its capability of giving insight into physical properties inaccessible to experiments or too difficult to be calculated from theoretical considerations. Needless to say, the effectiveness of MD is strictly bound to the availability of reliable models for the interparticle potentials. However, even the study of very simple model fluids, such as those ruled by hard-sphere (HS) [21-23], or Lennard-Jones (LJ) [3,24,25] interactions, has provided extremely useful results for the basic understanding of dynamical structures.

On the other hand, $S(Q, \omega)$ is the natural output of spectroscopic techniques based on the measurement of the (double-differential) cross-section in scattering experiments where energy $\hbar \omega$ and momentum $\hbar Q$ are transferred from the probe to the sample. Inelastic scattering of light, ultraviolet (UV) radiation, $\mathrm{x}$ rays, or thermal neutrons have been used for this purpose and, in the specific field of acoustic excitations, share nowadays the common denomination of "Brillouin scattering" that was initially coined for light scattering studies where the well-known Brillouin lines reflect the existence of propagating sound modes.

The $Q$ range of interest for the investigation of these phenomena goes usually from zero up to a value which corresponds to wavelengths of the order of the distance travelled between two consecutive collisions experienced by one atom. Thus, if $\ell$ is such a characteristic length (for 
example, the Enskog mean free path of an equivalent HS fluid [22]), the most frequently studied range is identified by the condition $0<Q \ell \sim 1$. With visible radiation, density fluctuations can only be explored at very low $Q$ (of the order of $0.01 \mathrm{~nm}^{-1}$ ), where hydrodynamics applies $[19,26]$. Non-hydrodynamic regimes can also be studied by means of light scattering, but this requires to increase $\ell$ by reducing the density to that of dilute gases $[27,28]$. At about the same time as for light Brillouin spectroscopy, neutron scattering also came into use in order to probe much higher $Q$ values, of the order of the position $Q_{\mathrm{p}}$ of the main peak of the static structure factor $S(Q)$, showing the persistence of collective excitations with wavelengths comparable with the typical microscopic distances of a dense fluid [29-31].

More recent technical advances are the extension of neutron scattering to lower $Q$ (down to $Q \sim 0.2 \mathrm{~nm}^{-1}$ ) [32-34], the advent of high-resolution x-ray scattering [35-37], widening the energy window at a given $Q$ due to the absence of the intrinsic kinematic limitations of neutron scattering, and, finally, the application of UV spectroscopy [38] to cover the $Q$ gap between light scattering and the other techniques.

The whole $Q$ range covered by a full set of experimental methods is thus very broad. Nevertheless, the theoretical developments that enable a detailed description of the measured spectral distributions are essentially the same at all the $Q$ values relevant to the investigation of sound excitations. Thus, a unitary picture has been established in this field.

In section 2 we summarize the basic theoretical concepts, described more in detail in recent works $[39,40]$, including the commonly used fit models and the criteria for a quantitative assessment of the excitation frequencies and damping. Sections 3, 4, and 5 are devoted to a short discussion of a few examples. The conclusions are contained in section 6 .

\section{Theoretical framework}

Here we give a short summary of the ideas and formulas relevant to the description of acoustic excitations in the simplest case of monatomic one-component fluids. We recall that we are dealing with classical systems for which $S(Q, \omega)$ is an even function of frequency at constant $Q$. This implies that, in applying theoretically derived or fit model line shapes to real experimental data, proper account must be taken of the detailed-balance asymmetry, besides the instrumental resolution broadening. The theoretical description of the acoustic excitations in fluids, in the greatest part of the published works, especially in recent years, is based on the use of the concept of memory function in the time evolution of the intermediate scattering function $F(Q, t)$. One then arrives at the basic Langevin-type equation [19]

$$
\ddot{F}(Q, t)+\int_{0}^{t} \mathrm{~d} t^{\prime} M\left(Q, t-t^{\prime}\right) \dot{F}\left(Q, t^{\prime}\right)+\left\langle\omega_{Q}^{2}\right\rangle F(Q, t)=0,
$$

where the dots denote time derivatives and $\left\langle\omega_{Q}^{k}\right\rangle$ is the $k$-th frequency moment of the normalized dynamic structure factor $I(Q, \omega)=S(Q, \omega) / S(Q)$. If (1) is solved in terms of the Laplace transforms of $F(Q, t)$ and $M(Q, t)$, denoted by $\tilde{F}(Q, z)$ and $\tilde{M}(Q, z)$ respectively, with $F(Q, 0)=S(Q)$ and $\dot{F}(Q, 0)=0$, one obtains

$$
\frac{\tilde{F}(Q, z)}{S(Q)}=\left(z+\frac{\left\langle\omega_{Q}^{2}\right\rangle}{z+\tilde{M}(Q, z)}\right)^{-1}
$$

and, from this, the general expression for the spectrum

$$
I(Q, \omega)=\frac{1}{\pi} \operatorname{Re} \frac{\tilde{F}(Q, \mathrm{i} \omega)}{S(Q)}=\frac{1}{\pi} \frac{\left\langle\omega_{Q}^{2}\right\rangle M^{\prime}(Q, \omega)}{\left[\omega^{2}-\left\langle\omega_{Q}^{2}\right\rangle+\omega M^{\prime \prime}(Q, \omega)\right]^{2}+\left[\omega M^{\prime}(Q, \omega)\right]^{2}},
$$

where $M^{\prime}(Q, \omega)$ and $M^{\prime \prime}(Q, \omega)$ are the real and imaginary part, respectively, of $\tilde{M}(Q, \mathrm{i} \omega)$.

Equation (3) would solve the problem fully, were it not for the fact that $M(Q, t)$ is actually unknown and cannot be determined from theoretical arguments. Therefore, realistic modelling of 
the memory effects becomes an essential step towards an accurate accounting of the measured or simulated line shapes. This has proved to be a very useful approach, because very simple expressions of $M(Q, t)$ can be shown to give rise to realistic forms of the spectral distribution $[19,39]$.

Various attempts to theoretically determine the time dependence of the memory function were made in the past mainly by testing different expressions against dynamics simulations [41,42]. It is a matter of fact, however, that in the greatest part of the works that appeared in the literature on the subject, $M(Q, t)$ is given the form of a $\delta$-function and/or a number of exponentials [39]. Then one can write $(3)$ in the form $\tilde{F}(Q, z) / S(Q)=V(z) / W(z)$, where $V$ and $W$ are polynomials in the complex variable $z$ having real, $Q$-dependent coefficients and degrees $p-1$ and $p$, respectively, if there are $p-2$ exponentials in $M(Q, t)$. If, for any $Q$, the $p$ zeros of $W(z)$ are labelled as $z_{j}$ (with $j=\mathrm{A}, \mathrm{B}, \ldots)$, one can also write

$$
\frac{\tilde{F}(Q, z)}{S(Q)}=\sum_{j} \frac{I_{j}(Q)}{z-z_{j}(Q)}
$$

where the complex amplitudes $I_{j}$ can be explicitly calculated [39] and satisfy the sum rule $\sum_{j} I_{j}=$ 1. In what follows we will omit the indication of the $Q$ dependence of $I_{j}$ and $z_{j}$. In the time domain, (4) is equivalent to $F(Q, t) / F(Q, 0)=\sum_{j} I_{j} \exp \left(z_{j} t\right)$, from which it is seen that the real part of all $z_{j}$ 's must be negative.

The existence of acoustic modes is easily related to the presence of two complex conjugate values among the $z_{j}$ 's for which we reserve the subscripts A and B, according to the sign of their imaginary part. Then, letting $z_{\mathrm{A}, \mathrm{B}}=-z_{\mathrm{s}} \pm \mathrm{i} \omega_{\mathrm{s}}$, with $\omega_{\mathrm{s}}>0$, and writing the corresponding amplitudes as $I_{\mathrm{A}, \mathrm{B}}=I_{\mathrm{S}}\left(1 \mp \mathrm{i} b_{\mathrm{s}}\right)$, the spectrum is given by

$$
I(Q, \omega)=\frac{1}{\pi}\left[\sum_{j=C, \mathrm{D} \ldots} \frac{-I_{j} z_{j}}{\omega^{2}+z_{j}^{2}}\right]+\frac{1}{\pi} I_{\mathrm{s}}\left[\frac{z_{\mathrm{s}}+b_{\mathrm{s}}\left(\omega+\omega_{\mathrm{s}}\right)}{\left(\omega+\omega_{\mathrm{s}}\right)^{2}+z_{\mathrm{s}}^{2}}+\frac{z_{\mathrm{s}}-b_{\mathrm{s}}\left(\omega-\omega_{\mathrm{s}}\right)}{\left(\omega-\omega_{\mathrm{s}}\right)^{2}+z_{\mathrm{s}}^{2}}\right],
$$

where the last two terms represent the Brillouin lines that are the spectral signature of the acoustic excitations of energy $\hbar \omega_{\mathrm{s}}$ (the subscript s standing for "sound"). The remaining terms are central lines building up the quasielastic part of the spectrum. All lines have a Lorentzian shape with halfwidths given by the negative of the real part of the various roots $z_{j}$ of the equation $W(z)=0$. The side lines, however, are distorted by the presence of asymmetric terms, related to the imaginary part of $I_{\mathrm{A}, \mathrm{B}}$ and deriving from the finiteness of $\left\langle\omega_{Q}^{2}\right\rangle$. The time correlation corresponding to the spectrum (5) is

$$
\frac{F(Q, t)}{F(Q, 0)}=\left[\sum_{j=\mathrm{C}, \mathrm{D} \ldots} I_{j} \exp \left(z_{j} t\right)\right]+2 I_{\mathrm{s}} \exp \left(-z_{\mathrm{s}} t\right) \frac{\cos \left(\omega_{\mathrm{s}} t-\varphi\right)}{\cos \varphi}
$$

with the sound modes appearing as one exponentially-damped oscillation with frequency $\omega_{\mathrm{s}}$ and a phase shift given by $\tan \varphi=b_{\mathrm{s}}$.

In the hydrodynamic situation (rigorously, in the limit case $Q \rightarrow 0$ ), where the fluid is probed as a continuum, $I(Q, \omega)$ is given by the Rayleigh-Brillouin $(\mathrm{RB})$ expression derived from linearization of the Navier-Stokes equations. Such a line shape can also be directly obtained from the assumption

$$
M(Q, t)=2 \nu Q^{2} \delta(t)+\left(\gamma_{0}-1\right)\left\langle\omega_{0}^{2}\right\rangle \exp \left(-\gamma_{0} D_{\mathrm{T}} Q^{2} t\right)
$$

where $\nu, D_{\mathrm{T}}$, and $\gamma_{0}$ are the kinematic longitudinal viscosity, the thermal diffusivity, and the specific-heat ratio, respectively, and $\left\langle\omega_{0}^{2}\right\rangle=k_{\mathrm{B}} T Q^{2} / m S(0)=c_{\mathrm{S}}^{2} Q^{2} / \gamma_{0}$ is the $Q \rightarrow 0$ limit of $\left\langle\omega_{Q}^{2}\right\rangle$, where $c_{\mathrm{S}}$ is the adiabatic sound velocity, $k_{\mathrm{B}}$ is the Boltzmann constant, $T$ is the temperature, and $m$ is the mass of the particles [19]. In this case $p=3$ and the spectrum $I(Q, \omega)$ is then given by (5) with only one central line, called the "heat" mode.

In the $Q$ range accessed by neutron or x-ray scattering, the RB theory, derived under the hydrodynamic condition of very-long-wavelength excitations, does not account for the measured spectral shapes. Therefore the observation of acoustic modes at such $Q$ values requires the models 
for $I(Q, \omega)$ to be valid in non-hydrodynamic regimes. These models have been customarily adopted as functions to be fitted to constant- $Q$ dynamic structure factor data, with parameters free to vary with the value of $Q$. At low enough $Q$, all models of $M(Q, t)$ are bound to reproduce the hydrodynamic behaviour, and thus display the Brillouin pair of lines due to the occurrence of two complex roots of $W(z)$. Here we list the most often used models by simply recalling their respective memory functions. More details on the properties of the various expressions can be found in [39].

The "generalized RB triplet" (GRB) model keeps the same expression of the RB line shape, with a memory function written as

$$
M(Q, t)=2 B(Q) \delta(t)+(\gamma(Q)-1)\left\langle\omega_{Q}^{2}\right\rangle \exp \left[-\Gamma_{\mathrm{T}}(Q) t\right]
$$

Another widely used model, called "viscoelastic" (VE), replaces the $\delta$-function with an exponential term, leading to

$$
M(Q, t)=\left[\omega_{\mathrm{L}}^{2}(Q)-\gamma(Q)\left\langle\omega_{Q}^{2}\right\rangle\right] \exp [-t / \tau(Q)]+(\gamma(Q)-1)\left\langle\omega_{Q}^{2}\right\rangle \exp \left[-\Gamma_{\mathrm{T}}(Q) t\right],
$$

with $p=4$. The various quantities appearing in (8) and (9) are unknown functions of $Q$ whose asymptotic behaviours in the limit $Q \rightarrow 0$ are to be in agreement with the exact RB theory. Moreover, in $(9), \omega_{\mathrm{L}}^{2}(Q)=\left\langle\omega_{Q}^{4}\right\rangle /\left\langle\omega_{Q}^{2}\right\rangle$, since the absence of the $\delta$-function in $M(Q, t)$ makes the fourth frequency moment of $I(Q, \omega)$ a finite quantity.

To the above mentioned models, one should also add those obtained from (8) and (9) by deleting the thermal part of the memory function, i.e., by letting $\gamma(Q)=\gamma_{0}=1$. Thus, one obtains, respectively, the "damped harmonic oscillator" (DHO), where $M(Q, t)$ reduces to the $\delta$-function term only $(p=2)$, and the "simple viscoelastic" (SVE) model introduced by Lovesey [43]

$$
M(Q, t)=\left[\omega_{\mathrm{L}}^{2}(Q)-\left\langle\omega_{Q}^{2}\right\rangle\right] \exp [-t / \tau(Q)]
$$

with $p=3$. Obviously, these simplified versions of the above models may only apply, at low $Q$, to the cases where $\gamma_{0}$ is close to unity, such as in some liquid metals, but the condition $\gamma(Q)=1$ can also occur at large $Q$ in other systems. An example of such a behaviour will be given in section 4 . At low $Q$, both DHO and SVE models produce the Brillouin doublet alone, without the central line due to thermal fluctuations. Actually, the spectrum corresponding to (10) does have a central line, corresponding to the so-called structural relaxation process described by the relaxation time $\tau(Q)$, but its amplitude can be shown to vanish in the $Q \rightarrow 0$ limit.

The fact that all the mentioned models give rise to spectra in the form (5) should be exploited to a larger extent than it has been done so far, and we emphasized in [39] the importance of using the parameters of (5), common to all models, rather than those of the memory functions, as fit parameters in order to compare the ability of different models to accurately reproduce the same spectral data. In $[39,40]$ we showed an example of how, in this way, it is possible to detect a transition that takes place, with increasing $Q$, from a hydrodynamic-like situation, well accounted for by the GRB model, to a viscoelastic regime.

Moreover, the common parametrization displayed in (5) has the important advantage of providing a full understanding of the dispersion curve of the acoustic excitations [39]. At each $Q$ the acoustic modes follow the dynamical behaviour of a damped harmonic oscillator with characteristic frequency $\Omega=\sqrt{z_{\mathrm{A}} z_{\mathrm{B}}}$ and damping coefficient $z_{\mathrm{s}}=-\left(z_{\mathrm{A}}+z_{\mathrm{B}}\right) / 2$ [40]. The propagation state of the excitation is then determined by whether such an oscillator finds itself in an under- or overdamping condition, with $\Omega>z_{\mathrm{s}}$ or $\Omega<z_{\mathrm{s}}$ respectively, or equivalently whether an oscillatory behaviour of $F(Q, t)$ takes place or not. In the first case, the right quantity to consider for a correct evaluation of the excitation frequency is $\omega_{\mathrm{s}}=\sqrt{\Omega^{2}-z_{\mathrm{S}}^{2}}$, which represents the frequency of the oscillating part of $F(Q, t)$ (see $(6))$. For overdamped excitations, instead, $\omega_{\mathrm{s}}=0, z_{\mathrm{A}}$ and $z_{\mathrm{B}}$ become real, and the spectral shape is modified by the transformation of the Brillouin lines into Lorentzians of half widths $-z_{\mathrm{A}}$ and $-z_{\mathrm{B}}$ centered at zero frequency and superimposed onto the quasielastic lines.

Thus the comparison between $\Omega$ and $z_{\mathrm{s}}$ provides a rigorous answer to the question of what is the propagation $Q$-range of acoustic excitations. Apart from qualitative considerations, the only other quantitative criterion found in the literature $[19,43]$ is based on the properties of the SVE model 
line shape, and corresponds to the condition of the existence of two relative maxima of $I(Q, \omega)$ at frequencies different from zero in addition to the presence of a peak at $\omega=0$. This requirement leads to the inequality

$$
\omega_{\mathrm{L}}^{2}(Q)>\frac{1}{\tau(Q)} \sqrt{2\left(2 \omega_{\mathrm{L}}^{2}(Q)-3\left\langle\omega_{Q}^{2}\right\rangle\right)-\frac{1}{\tau^{2}(Q)}}
$$

although a more frequently adopted criterion is the simpler one, not involving $\tau(Q)$, given by

$$
\omega_{\mathrm{L}}^{2}(Q)<3\left\langle\omega_{Q}^{2}\right\rangle
$$

which actually expresses a sufficient but not necessary condition for the existence of a three-peak spectrum. In other words, acoustic modes are said to be propagating if they appear as side peaks (though possibly largely broadened), but are defined as overdamped, and hence non-propagating, when they look like bumps, or shoulders, that do not give rise to relative maxima of the spectral intensity. Such a criterion is not rigorous and is by far too stringent, since in any case such broad features are located in frequency bands spread around non-zero values and having therefore a genuine inelastic character. Moreover, (11) has other drawbacks. First of all, it applies only to the SVE case, and cannot be easily generalized to the other line shape models. Moreover, it is based on the idea that the damping state of an excitation can depend on its strength. The above mentioned close mathematical analogy with a harmonic oscillator shows instead [40] that the amplitude of the sound mode has nothing to do with its being under- or overdamped.

It is to be noted that another argument based on the analysis of intensities rather than frequencies provides the most commonly applied method for the determination of the dispersion curve, namely the use of the maxima in the spectra of the longitudinal current correlation. While the use of (11) tends to an underestimation of the propagation $Q$ range, the application of this criterion, which does not have a theoretical justification, has in some sense an opposite effect, since it always provides a non-zero value for the excitation frequency even when the acoustic modes are truly overdamped $\left(\omega_{\mathrm{s}}=0\right)$, thus preventing the detection of $Q$-ranges for which the acoustic waves do not possibly propagate. This last remark also applies to other incorrect determinations of the excitation frequency, such as those based on the use of $\Omega$ or of $\sqrt{\gamma(Q)\left\langle\omega_{Q}^{2}\right\rangle}[39]$.

\section{Rare gases}

As applications of the concepts outlined in section 2, we briefly recall and discuss in the next three sections a few typical results for the collective acoustic dynamics in very simple fluids.

Argon is the prototype of a monatomic, classical, non-conductive fluid, and has been the object of many investigations. Moreover, it is an exceptionally convenient sample for neutron studies due to the very large, totally coherent, scattering cross-section of the ${ }^{36} \mathrm{Ar}$ isotope. Well-known pioneering neutron works on the collective dynamics of argon are, among others, those of references $[29,30]$ in the liquid and [44] in the compressed gas phase, while [45] reports on a light scattering experiment. Though sound modes were in some cases detected, depending on the investigated $Q$ range, and obviously with particular evidence in the light Brillouin case where $Q=0.017 \mathrm{~nm}^{-1}$ (see figure 3 of [45]), the first quantitative analysis of acoustic excitations was carried out in the neutron study of reference [46].

The generalized hydrodynamic model (8) (derived from kinetic-theoretical considerations) was fitted to constant- $Q$ spectra in an extended wavevector range $\left(4<Q / \mathrm{nm}^{-1}<38\right)$. Asymmetric Brillouin lines were detected, with one real and two complex conjugate poles of $\tilde{F}(Q, z)$, in the whole $Q$ range except for $17<Q / \mathrm{nm}^{-1}<21$, where a better fit was obtained with three real roots. The authors interpreted this result as the first observation in a real fluid of a "propagation gap" of sound modes, a phenomenon previously discovered in a HS system [22] and in a GRB analysis of a simulated LJ dense fluid [3].

In view of the discussion of the previous section, one can easily interpret the occurrence of such a gap as the one due to the transition from the under- to overdamping condition of the 
equivalent harmonic oscillator, with $\Omega<z_{\mathrm{s}}$, shortly after followed by a reverse transition restoring the propagating regime. However, the conclusions of reference [46] were criticized [47] on the ground that it may not be justifiable to speak of collective excitations when the spectral shape does not show visible inelastic structures, in agreement with the meaning of criterion (11). Apart from similar results obtained in other thermodynamic states of liquid argon and in liquid neon [48], where a propagation gap was also reported, this issue has not been dealt with in later works on fluid systems, because in the $Q$ range where a gap may possibly occur, dynamical data have been customarily analyzed with models not having $\omega_{\mathrm{s}}$ and $z_{\mathrm{s}}$ as explicit parameters. We will, however, come back to this point in section 4 .

Acoustic excitations of argon were also studied in the gas phase [33] at room temperature and much lower densities than in the liquid. Low values of $Q \ell$ (in the range $0.1 \leqslant Q \ell \leqslant 1$ ) could then be attained with small-angle inelastic scattering of cold neutrons. The GRB model was well fitted to the data, which is in close agreement with MD results [49]. At the lowest $Q \ell$ values, the spectra agree with the hydrodynamic RB line shape, while they begin to deviate from it with increasing $Q$ and/or decreasing density. Thus, the onset of non-hydrodynamic behaviour could be evidenced. From figure 1, where we display $\Omega, z_{\mathrm{s}}$ and $\omega_{\mathrm{s}}$ as obtained from the GRB fit to the 200 bar data, a slightly stronger upward curvature of $\Omega(Q)$ and a slightly smaller damping $z_{\mathrm{s}}$ are found with respect to the predictions of RB theory. Then $\omega_{\mathrm{s}}=\sqrt{\Omega^{2}-z_{\mathrm{s}}^{2}}$ stays above the RB dispersion curve, whose downward bending is effectively compensated, and a linear $Q$-dependence of $\omega_{\mathrm{s}}$ is found. This example also shows that the difference between $\Omega, \omega_{\mathrm{s}}$, and other frequency parameters should be taken into account if the presence or absence of the so-called positive dispersion has to be correctly assessed.

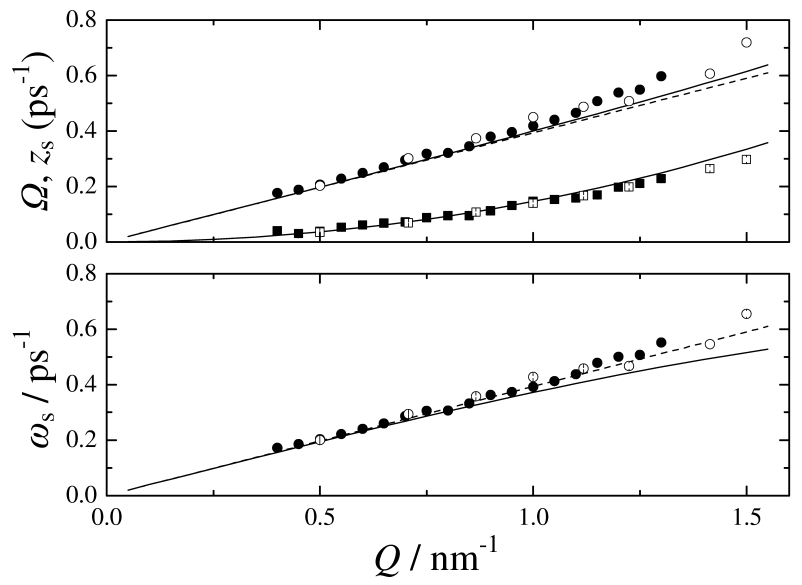

Figure 1. GRB analysis of neutron (full symbols) and MD (empty symbols) data on gaseous ${ }^{36} \mathrm{Ar}$ at 200 bar $[33,49]$. Solid lines are the corresponding calculations with RB theory. Upper frame: $\Omega$ (circles) and $z_{\mathrm{s}}$ (squares). Lower frame: $\omega_{\mathrm{s}}$ (circles). In both frames, the straight line $c_{\mathrm{s}} Q$ is shown by dashes.

Besides argon, neon has also been the object of studies for the collective dynamics. Here too, neutron [31,50] and light [45] scattering experiment can be traced back far in the past. More recently, as mentioned above, liquid neon was investigated in parallel with the neutron experiment on argon [48], and an overall similarity of the dynamical behaviour of the two liquids was found (see figure 12 of [48]), so that a corresponding-state principle was claimed to hold.

Neon has also been extensively studied by means of inelastic x-ray scattering. Experimental data in the room-temperature gas, at a pressure of $0.3 \mathrm{GPa}$ and a density close to that of the liquid phase, were found in agreement with LJ-simulated spectra, whose collective modes were then analyzed in terms of the GRB model [51] in the range $1<Q / \mathrm{nm}^{-1}<25$, showing the absence of positive dispersion. This marks a difference with respect to the liquid [48], though the densities are similar.

Later, more x-ray data at a number of thermodynamic states of gaseous and liquid neon were 
analyzed using, instead, the SVE model [52]. Here, a positive dispersion present in the liquid also tends to disappear in approaching supercritical temperatures. However, since the fitted model was not expressed in the form (5), the excitation frequencies were estimated through the use of various parameters different from $\omega_{\mathrm{s}}$, preventing the possibility of a comparison with previous studies or other fluids.

We recall from [39] that, if any of the model shapes (5) can be well fitted to the data, a positive dispersion can be attributed to either an initial decrease of $S(Q)$ at low $Q$ or to a reduction, with increasing $Q$, of the central line-width(s) with respect to the values given by the lowest order in a $Q$-power series expansion. Such a damping reduction, expressed by the renormalization function $r(Q)$ defined in $[39,40]$, plays an evident role in shaping the dispersion curve, and the explanation of its connection with the microscopic interactions would be of high interest. Unfortunately, this property of fluid systems is still to be adequately investigated.

\section{Methane}

Notwithstanding the obvious differences between monatomic and molecular systems, methane behaves very similarly to the rare-gas fluids if the centre-of-mass dynamics is considered, as it will be shown below. The comparison between neutron scattering [53] and simulation data has recently provided the possibility of selecting one among different site-site interaction potentials for methane [54], and, therefore, of validating MD results for the experimentally inaccessible partial carbon-carbon dynamic structure factor $S_{\mathrm{CC}}(Q, \omega)$, which is the molecular analogue of $S(Q, \omega)$ of monatomic systems. The centre-of-mass collective motions of liquid $\mathrm{CD}_{4}$ were then analyzed in a very wide $Q$ range (up to $Q \sim 3.7 Q_{\mathrm{p}}$ ) [40].

For $2<Q / \mathrm{nm}^{-1}<15\left(\sim 0.8 Q_{\mathrm{p}}\right)$ we applied both the GRB and VE fit models, obtaining practically indistinguishable results for $Q$ smaller than about $5 \mathrm{~nm}^{-1}$, while at higher $Q$ the presence of two quasielastic lines in the VE fit function permits a more accurate description of the central peak [39]. The viscoelastic model turns out to account well for the simulated spectral shape at larger $Q$ as well, up to $Q=67 \mathrm{~nm}^{-1}$. However, for $Q>37.5 \mathrm{~nm}^{-1}$, the thermal contribution to the dynamics vanishes, and the simpler viscoelastic model (SVE) becomes sufficient.

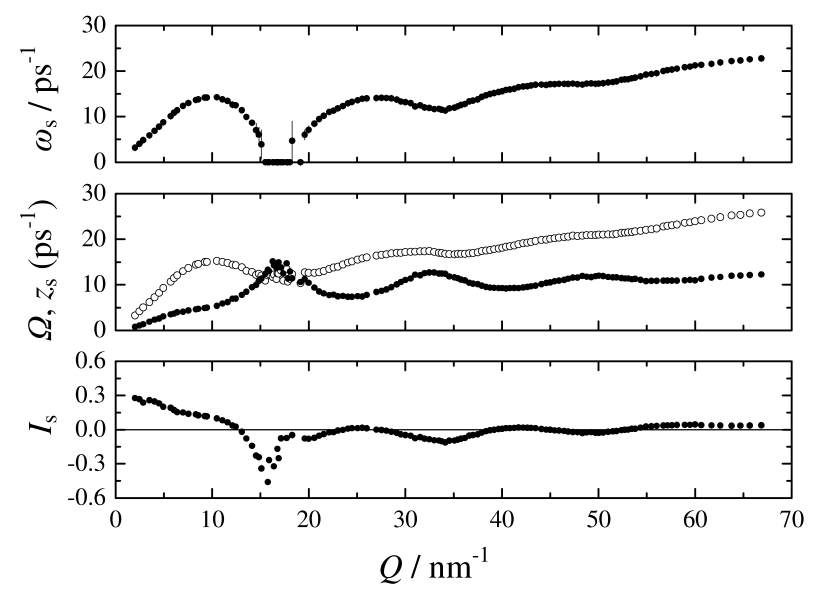

Figure 2. VE analysis of MD data for the centre-of-mass $S_{\mathrm{CC}}(Q, \omega)$ of liquid $\mathrm{CD}_{4}$ [40]. Upper frame: $\omega_{\mathrm{s}}$; middle frame: $\Omega$ (circles) and $z_{\mathrm{s}}$ (full dots); lower frame: $I_{\mathrm{s}}$ (full dots). For $Q>$ $37.5 \mathrm{~nm}^{-1}$ the SVE model (with $\gamma=1$ ) has been used.

The shape of the dispersion curve, reported in figure 2, shows a strong similarity to that of liquid argon [46]. In particular, methane also displays a propagation gap in a narrow $Q$ interval around $Q_{\mathrm{p}}$. Such a transition to an overdamped regime is clearly related to the $Q$ dependence of $\Omega$ and $z_{\mathrm{s}}$, and, in particular, to the decrease of $\Omega$, caused by the presence of a high peak in $S_{\mathrm{CC}}(Q)$, in coincidence with an increased damping. However, $\Omega$ becomes only slightly smaller than $z_{\mathrm{s}}$, so 
that only a weak overdamping is obtained. This fact suggests that an arrest of the propagation of sound modes can only occur in strongly structured fluids. Indeed, the whole $Q$-dependence of all the quantities plotted in figure 2 directly reflects the oscillatory behaviour of $S_{\mathrm{CC}}(Q)$. This shows that a strong relationship exists at all $Q$ values between the whole dynamics and the structural correlations. Figure 2 also shows that the amplitude $I_{\mathrm{S}}$ of the acoustic part of the intermediate scattering function rapidly decays towards zero for $Q$ larger than $Q_{\mathrm{p}}$ [40]. One can then conclude that acoustic excitations are present with a propagative nature up to very high $Q$, where their relevance is limited by a fading intensity rather than by a vanishing frequency.

\section{Rare-gas mixtures}

Rare-gas fluids have also played an important role in the investigation of a phenomenon which characterizes the collective dynamics of binary mixtures, namely the existence of the so-called fast and slow sounds. In the hydrodynamic regime, such systems behave as homogeneous fluids and display a regular acoustic mode propagating with the adiabatic sound velocity of the mixture. At higher $Q$, a dynamical decoupling of the two components takes place, more marked for higher mass ratios between the two species. A high-frequency propagation is supported by the light atoms only, while the heavy ones are incapable of following the fast oscillatory motion. On the other hand, a slow propagation is attributed to oscillations of the heavy particles.

Fast sound in gas mixtures was predicted to be observable through neutron scattering at intermediate densities [55,56], and through light scattering in the dilute case [57]. The presence of a fast sound is related to the existence of acoustic excitations in the partial dynamic structure factor of the light component but not in that of the heavy one. Then the visibility of the effect in the total $S(Q, \omega)$ depends on the concentrations of the two species and on their cross-sections relevant to the employed spectroscopic technique (scattering lengths for neutron and polarizabilities for light scattering).

Several studies were devoted to the exploration of these excitations. Considering only the most studied case of HeNe mixtures with helium concentration ranging from 0.65 to 0.80 and total number densities around $15 \mathrm{~nm}^{-3}$, the first neutron observation of fast sound [58,59] showed for $Q>4 \mathrm{~nm}^{-1}$ an excitation propagating with a speed very close to that of pure helium at the same total density. MD simulations [60] and theoretical calculations $[61,62]$ were also carried out on the same system. Nevertheless, the question of how this fast sound mode is connected to the hydrodynamic one has eluded for some time the efforts of the investigators. Based on the kinetic theory it was predicted [59] that the fast branch would disappear at $Q \sim 0.7 \mathrm{~nm}^{-1}$, in agreement with MD results [60]. However, a later neutron experiment showed a linear dispersion with the hydrodynamic sound speed up to $Q=1.8 \mathrm{~nm}^{-1}[63]$, so that the $Q$ range where the transition from hydrodynamic to fast sound takes place was identified with the still unexplored interval between those spanned by the two neutron measurements. Such a transition, shown in figure 3, later turned out to be a smooth one in an MD simulation in agreement with both experimental data sets [64]. The same simulation did not show evidence of a slow sound, which, on the contrary, appears clearly at a much higher density.

It should also be mentioned that the detection and the correct location of inelastic mode frequencies in the spectra of fluid mixtures is even more dependent than in pure systems on the adoption of adequate criteria for the assignment of the excitation energies. A clear example of this problem is given in [64], where it was shown that the identification of mode frequencies with the position of maxima in the Ne-Ne partial longitudinal-current spectra leads to a wrong dispersion curve for the slow sound.

We finally note that, in the case of mixtures, the extension of the GRB line shape to the two-component systems is practically the only model applied so far to the description of collectivedynamics spectra, and only little work has been done $[65,66]$ in order to define or test other spectral models. 


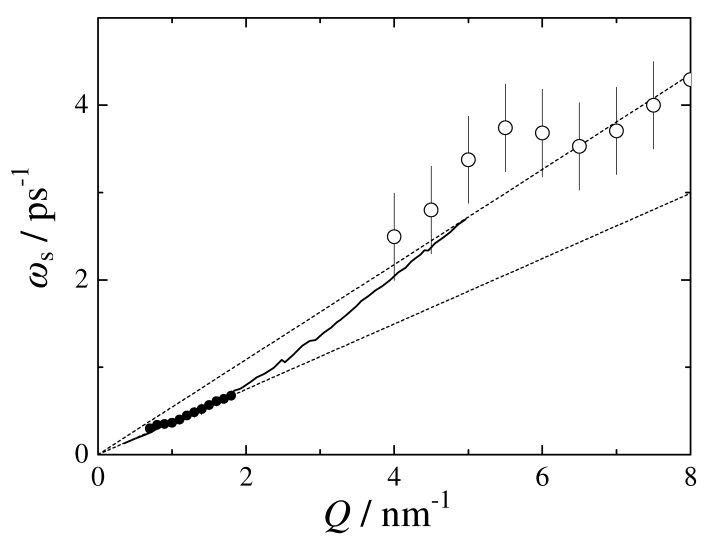

Figure 3. Dispersion curve of the acoustic mode in a HeNe gaseous mixture. The neutron results from [63] (full dots) and from [58] (circles) are shown together with MD data from [64] (solid line). The straight lines corresponding to the sound speed of the mixture $\left(c_{\mathrm{s}}=374 \mathrm{~m} / \mathrm{s}\right)$ and of pure helium $\left(c_{\mathrm{s}}=544 \mathrm{~m} / \mathrm{s}\right)$ are shown as dashes.

\section{Conclusions}

Even a quick glance at the very recent literature on the subject of collective modes in fluid systems is sufficient to realize that this research field is a very active one. New classes of liquids have come under the scrutiny of experimentalists who take advantage of impressive advances in the capabilities of innovative instrumentation and techniques and in the computing power available for simulation studies. Besides the traditionally investigated rare-gas fluids and liquid metals, growing attention has been paid to molecular liquids, liquid alloys, metal vapours, molten salts and oxides, metals in solutions, and complex macromolecular systems.

From the viewpoint of the analysis and the interpretation of collective-dynamics spectra, progress has also been made, with the refinement of model line shapes allowing for more detailed descriptions of the dynamical behaviour in wide energy and momentum ranges, and with the definition of rigorous criteria for the quantitative assessment of the nature of collective acoustic modes.

Due to such advances, reliable tools are nowadays available for a full characterization of the collective excitations in a large variety of fluids. It turns out to be a quite general result that the whole spectral distribution is essentially determined by the microscopic damping processes that take place in the fluid and shape both the quasielastic and the propagating modes [39]. One can thus envisage, as a further step in the understanding of these processes, the direct tackling of a far more fundamental question, namely the deep connection between the emergence of such a dynamical behaviour and the underlying microscopic forces among the individual particles. The way in which the details of the potential functions affect the $Q$ dependence of the various relaxation mechanisms associated to collective motions is still very little investigated and deserves dedicated efforts. In this sense, the study of very simple fluids such as rare gases and simple molecules is likely to still have much to reveal.

\section{References}

1. Kambayashi S., Hiwatari Y., Phys. Rev. E, 1994, 49, 1251.

2. Anento N., Padró J.A., Canales M., J. Chem. Phys., 1999, 111, 10210.

3. de Schepper I.M., van Rijs J.C., van Well A.A., Verkerk P., de Graaf L.A., Bruin C., Phys. Rev. A, 1984, 29, 1602.

4. Canales M., Padró J.A., Phys. Rev. E, 1999, 60, 551.

5. Maitland G.C., Rigby M., Smith E.B., Wakeham W.A., Intermolecular Forces, their Origin and Determinations, Clarendon, Oxford, 1981.

6. March N., Liquid Metals: Concepts and Theory, 1990, Cambridge University Press, Cambridge. 
7. Scopigno T., Ruocco G., Sette F., Rev. Mod. Phys., 2005, 77, 881.

8. Glyde H.R., Excitations in Liquid and Solid Helium, 1994, Clarendon, Oxford.

9. Griffin A., Excitations in a Bose-Condensed Liquid, 1993, Cambridge University Press, Cambridge.

10. Bermejo F.J., Fåk B., Bennington S.M., Fernández-Perea R., Cabrillo C., Dawidowski J., FernándezDiaz M.T., Verkerk P., Phys. Rev. B, 1999, 60, 15154.

11. Bermejo F.J., Kinugawa K., Cabrillo C., Bennington S.M., Fåk B., Fernández-Díaz M.T., Verkerk P., Dawidowski J., Fernández-Perea R., Phys. Rev. Lett., 2000, 84, 5359.

12. Bermejo F.J., Mompeán F.J., García-Hernández M., Martínez J.L., Martin-Marero D., Chahid A., Senger G., Ristig M.L., Phys. Rev. B, 1993, 47, 15097.

13. Carneiro K., Nielsen M., McTague J.P., Phys. Rev. Lett., 1973, 30, 481.

14. Cunsolo A., Colognesi D., Sampoli M., Senesi R., Verbeni R., J. Chem. Phys., 2005, 123, 114509.

15. García-Hernández M., Mompeán F.J., Schärpf O., Andersen K.H., Fåk B., Phys. Rev. B, 1999, 59, 958.

16. Mompeán F.J., García-Hernández M., Fåk B., Phys. Rev. B, 1997, 56, 11604.

17. Mukherjee M., Bermejo F.J., Fåk B., Bennington S.M., Europhys. Lett., 1997, 40, 153.

18. van Kranendonk J., Solid Hydrogen, Plenum, New York, 1983.

19. Balucani U., Zoppi M., Dynamics of the Liquid State, Clarendon, Oxford, 1994.

20. Allen M.P., Tildesley D.J., Computer Simulations of Liquids, Clarendon, Oxford, 1987.

21. Alley W.E., Alder B.J., Phys. Rev. A, 1983, 27, 3158.

22. Kamgar-Parsi B., Cohen E.G.D., de Schepper I.M., Phys. Rev. A, 1987, 35, 4781.

23. Sharma R.K., Tankeshwar K., Pathak K.N., Ranganathan S., Johnson R.E., Phys. Rev. E, 1997, 55, 1550 .

24. Enciso E., Almarza N.G., del Prado V., Bermejo F.J., López Zapata E., Ujaldón M., Phys. Rev. E, 1994, 50, 1336.

25. Mryglod I.M., Omelyan I.P., Tokarchuk M.V., Mol. Phys., 1995, 84, 235.

26. Boon J.P., Yip S., Molecular Hydrodynamics, McGraw-Hill, New York, 1980.

27. Clark N. A., Phys. Rev. A, 1975, 12, 232.

28. Ghaem-Maghami V., May A.D., Phys. Rev. A, 1980, 22, 698.

29. Sköld K., Larsson K.E., Phys. Rev., 1967, 161, 102.

30. Sköld K., Rowe J.M., Ostrowski G., Randolph P.D., Phys. Rev. A, 1972, 6, 1107.

31. Bell H., Moeller-Wenghoffer H., Kollmar A., Stockmeyer R., Springer T., Stiller H., Phys. Rev. A, $1975, \mathbf{1 1}, 316$.

32. Egelstaff P.A., Kearley G., Suck J.-B., Youden J.P.A., Europhys. Lett., 1989, 10, 37.

33. Bafile U., Verkerk P., Barocchi F., de Graaf L.A., Suck J.-B., Mutka H., Phys. Rev. Lett., 1990, 65, 2394.

34. Aisa D. et al., Nucl. Instrum. Meth. Phys. Res. A, 2005, 544, 620.

35. Dorner B., Peisl H., Nucl. Instrum. and Meth., 1983, 208, 587.

36. Burkel E., Sinn H., J. Phys.: Condens. Matter, 1994, 6, A225.

37. Masciovecchio C., Bergmann U., Krisch M., Ruocco G., Sette F., Verbeni R., Nucl. Instrum. Meth. Phys. Res. B, 1996, 111, 181.

38. Masciovecchio C., Gessini A., Santucci S.C., J. Non-Cryst. Solids, 2006, 352, 5126.

39. Bafile U., Guarini E., Barocchi F., Phys. Rev. E, 2006, 73, 061203.

40. Sampoli M., Bafile U., Barocchi F., Guarini E., Venturi G., J. Phys.: Condens. Matter, 2008, 20, 104206.

41. Ailawadi N.K., Rahman A., Zwanzig R., Phys. Rev. A, 1971, 4, 1616.

42. Barker M.I., Gaskell T., J. Phys. C: Solid State Phys., 1972, 5, 3279.

43. Lovesey S.W., J. Phys. C: Solid State Phys., 1971, 4, 3057.

44. Postol T.A., Pelizzari C.A., Phys. Rev. A, 1978, 18, 2321.

45. Fleury P.A., Boon J.P., Phys. Rev., 1969, 186, 244.

46. de Schepper I.M., Verkerk P., van Well A.A., de Graaf L.A., Phys. Rev. Lett., 1983, 50, 974.

47. Lovesey S.W., Phys. Rev. Lett., 1984, 53, 401.

48. van Well A.A., de Graaf L.A., Phys. Rev. A, 1985, 32, 2396.

49. Bafile U., Barocchi F., Neumann M., Verkerk P., J. Phys.: Condens. Matter, 1994, 6, A107.

50. Buyers W.J.L., Sears V.F., Lonngi P.A., Lonngi D.A., Phys. Rev. A, 1975, 11, 697.

51. Cunsolo A., Pratesi G., Ruocco G., Sampoli M., Sette F., Verbeni R., Barocchi F., Krisch M., Masciovecchio C., Nardone M., Phys. Rev. Lett., 1998, 80, 3515.

52. Cunsolo A., Pratesi G., Verbeni R., Colognesi D., Masciovecchio C., Monaco G., Ruocco G., Sette F., J. Chem. Phys., 2001, 114, 2259. 
53. Guarini E., Bafile U., Barocchi F., Demmel F., Formisano F., Sampoli M., Venturi G., Europhys. Lett., 2005, 72, 969.

54. Guarini E., Sampoli M., Venturi G., Bafile U., Barocchi F., Phys. Rev. Lett., 2007, 99, 167801.

55. Campa A., Cohen E.G.D., Phys. Rev. Lett., 1988, 61, 853.

56. Montfrooij W., Westerhuijs P., de Schepper I., Phys. Rev. Lett., 1988, 61, 2155.

57. Campa A., Cohen E.G.D., Phys. Rev. A, 1989, 39, 4909.

58. Montfrooij W., Westerhuijs P., de Haan V.O., de Schepper I.M., Phys. Rev. Lett., 1989, 63, 544.

59. Westerhuijs P., Montfrooij W., de Graaf L.A., de Schepper I.M., Phys. Rev. A, 1992, 45, 3749.

60. Enciso E., Almarza N.G., Domínguez P., González M.A., Bermejo F.J., Phys. Rev. Lett., 1995, 74, 4233.

61. Bryk T., Mryglod I., Kahl G., Phys. Rev. E, 1997, 56, 2903.

62. Bryk T., Mryglod I., Condens. Matter Phys., 2004, 7, 15.

63. Bafile U., Verkerk P., Guarini E., Barocchi F., Phys. Rev. Lett., 2001, 86, 1019.

64. Sampoli M., Bafile U., Guarini E., Barocchi F., Phys. Rev. Lett., 2002, 88, 085502.

65. Jäckle J., Pieroth M., J. Phys.: Condens. Matter, 1990, 2, 4963.

66. Anento N., González L.E., González D.J., Chushak Y., Baumketner A., Phys. Rev. E, 2004, 70, 041201.

\title{
Колективна динаміка в благородних газах та інших дуже простих класичних плинах
}

\author{
У.Бафіле ${ }^{1}$, Ф.Бароккі ${ }^{2,3}$, Е.ґуаріні ${ }^{2,3}$ \\ 1 Інститут складних систем, Національна Рада Досліджень, Сесто Фіорентіно, Італія \\ 2 Фізичний факультет, Університет Флоренції, Сесто Фіорентіно, Італія \\ 3 Національна Рада Досліджень, Центр SOFT п/а Фізичний факультет, Університет Флоренції, Сесто \\ Фіорентіно, Італія \\ Отримано 12 листопада 2007 р.
}

\begin{abstract}
Інертні гази та їхні рідини є найпростішими системами для точних досліджень колективної динаміки плинів. Було пророблено значну роботу з використанням різноманітних спектроскопічних методик, моделювання методом молекулярної динаміки та теоретичних розробок для розуміння мікроскопічних процесів, що приймають участь у поширенні звукових збуджень у газах та рідинах. Ми подаємо короткий огляд інтерпретаційних схем, що застосовуються для опису таких збуджень, та нагадуємо декілька результатів, отриманих із аналізу плинів інертних газів та інших дуже простих систем.
\end{abstract}

Ключові слова: динаміка плинів, колективні збудження

PACS: $61.20 . L C, 61.20 . G y, 61.25 . B i$ 